\title{
Wie Innovation direkt von der Basis her gefördert wird
}

\section{Felix Helg}

Dr. phil., Inhaber und Geschäftsführer Helg Consulting, Projektleiter "Brennpunkte Gesundheit Thurgau»

In einem zweijährigen Prozess ist es im Kanton Thurgau gelungen, dank Vernetzung vieler Leistungserbringer und Stakeholder im Gesundheitswesen drei Projekte durchzuführen. Das Projekt «Brennpunkte Gesundheit Thurgau» leistet einen wichtigen Beitrag zur Optimierung des Thurgauer Gesundheitswesens und für die Vernetzung der beteiligten Stakeholder.

\section{Die Ausgangslage}

«Der Krieg ist der Vater aller Dinge.» Wie schon Heraklit wusste, stehen Auseinandersetzung und Konflikt oft am Anfang von kreativen Prozessen. Dies war auch im Projekt «Brennpunkte Gesundheit Thurgau» der Fall. Im Zusammenhang mit der Einführung der Fallpauschalen im stationären Bereich kam es zu Spannungen zwischen einzelnen Leistungserbringern im kantonalen Gesundheitswesen. An einem runden Tisch unter der Leitung des damaligen Gesundheitsdirektors konnten die Spannungen besprochen werden. Um den Erfolg dieses runden Tisches nachhaltig zu verankern, erhielt die Ärztegesell-

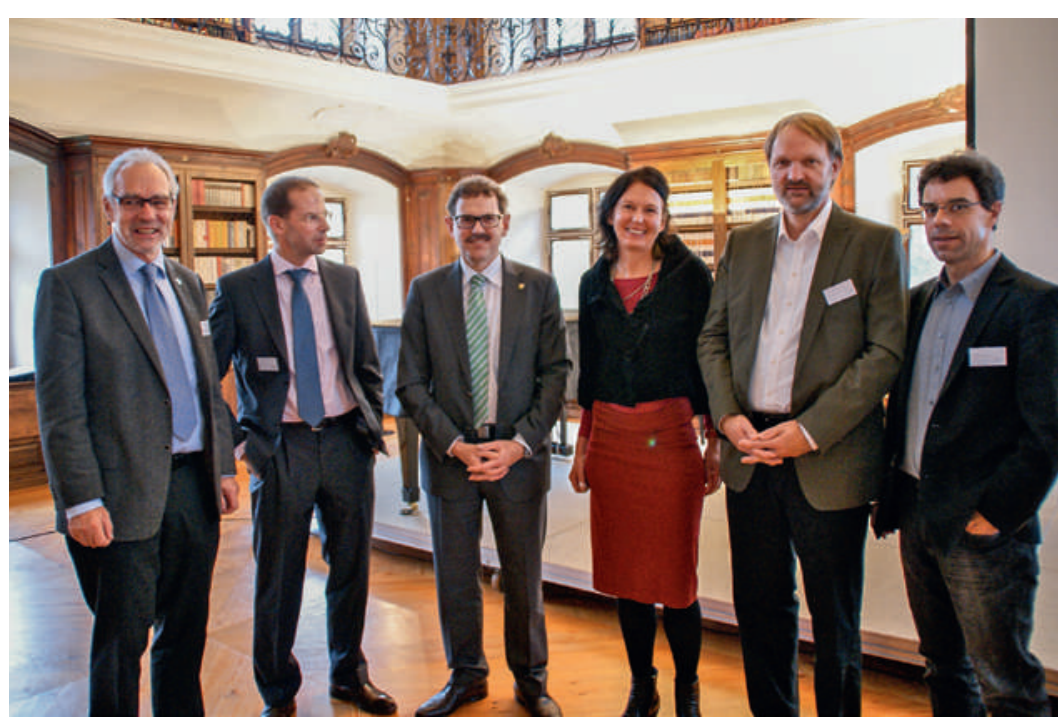

Einige Projekt-Teilnehmer (v.I.n.r.): Kantonsarzt Olivier Kappeler, Projektleiter Felix Helg, Regierungsrat Jakob Stark, Karin Frick, Gottlieb Duttweiler Institut, Präsident Ärztegesellschaft Daniel Jud, CFO Spital Thurgau Peter Heri an der Tagung im Kloster Fischingen.
«Brennpunkte Gesundheit Thurgau» -

Encourager l'innovation depuis la base

En l'espace de deux ans, le canton de Thurgovie est parvenu à développer et à finaliser trois projets de différents niveaux de complexité grâce à la mise en réseau de nombreux prestataires de soins et acteurs de la santé.

Dans un premier temps, les quelque cent acteurs de la santé impliqués ont, à l'occasion d'un congrès, élaboré plusieurs ébauches de projet dans le but de contribuer à améliorer le système de santé cantonal. Puis, au cours des 18 mois suivants, trois idées ont été approfondies. Le premier projet vise à sensibiliser les supérieurs et les responsables du personnel à la manière de réagir face à des collaborateurs souffrant de problèmes psychiques. Le deuxième projet présente quant à lui des propositions pour garantir le bon fonctionnement de la prise en charge des patients dans le canton. Quant au troisième projet, intitulé "eMediplan», il propose un concept en vue d'un plan électronique des médicaments.

Le projet "Brennpunkte Gesundheit Thurgau» a réuni des professionnels du domaine de la santé qui ne se seraient jamais rencontrés autrement, mettant ainsi en évidence l'importance de la collaboration pour affronter les enjeux complexes à venir. Fortes de cette réussite, de nombreuses organisations impliquées ont exprimé leur intention de soutenir activement la reconduction de ce projet. 
schaft des Kantons Thurgau vom Departement für Finanzen und Soziales den Auftrag, «unter Einbezug der Leistungserbringer der gesamten Behandlungskette [...] sowie weiterer Stakeholder (Verband Thurgauer Gemeinden, Verband Thurgauer Schulgemeinden, Industrie- und Handelskammer, Gewerbeverband, u.a.) dem Chef des Departements für Finanzen und Soziales drei Projektvorschläge [...] zu aktuellen Vor- und Versorgungsplanungsthemen im Kanton Thurgau vorzulegen» (Auszug aus dem Projektauftrag).

In einem weiteren Schritt sollen dem Regierungsrat des Kantons Thurgau Vorschläge zur Zusammensetzung und zur Arbeitsweise eines möglichen Versorgungsrates gemacht werden.

\section{Der Prozess}

Um den Auftrag des Departementvorstehers zu erfüllen, setzt die Ärztegesellschaft eine Projektgruppe ein. Diese einigt sich auf folgendes Vorgehen: An einer Tagung, zu der die wichtigsten Stakeholder des kantonalen Gesundheitswesens eingeladen werden, sollen drei Projektskizzen ausgewählt werden, für die in der Folge ein Konzept erarbeitet wird.

An einem schönen Sommertag im Juni 2013 finden sich über 100 Personen in der Kartause Ittingen ein. Mit der Methode Open Space wird während des ganzen Tages intensiv gearbeitet - am Schluss sind über 20 Projektideen formuliert, die dem kantonalen Gesundheitswesen frische Impulse verleihen sollen. Jeder Teilnehmer, jede Teilnehmerin erhält Gelegenheit, eine Idee für ein innovatives Projekt zu nennen. Finden sich genügend weitere Interessenten, die diesen Gedanken vertiefen wollen, so entsteht im Verlauf des Tages eine Skizze, die den übrigen Anwesenden vorgestellt wird. Am Schluss der Veranstaltung bestimmen die Teilnehmenden gemeinsam, welche Ideen am meisten Anklang finden.

Der Open-Space-Ansatz zeichnet sich dadurch aus, dass die Erarbeitung der Projektskizzen in grosser Freiheit und Eigenverantwortung geschieht. Ich kann als Teilnehmer dort mitwirken, wo ich das sinnvoll finde. Wenn ich das Gefühl habe, an einem anderen Ort mehr zum Erfolg beizutragen, verlasse ich den Workshop und beteilige mich an einer anderen Diskussionsrunde. Open Space basiert auf zwei Grund-

Projektgruppe

Felix Helg, Projektleiter; Daniel Jud, Präsident Ärztegesellschaft Thurgau; Olivier Kappeler, Kantonsarzt und Chef Amt für Gesundheit Thurgau; Peter Heri, CFO Spital Thurgau AG; Christa Lanzicher, Geschäftsführerin Spitex Verband Thurgau; Christa Thorner, Kantonsrätin, Präsidentin Perspektive Thurgau gedanken: dem Prinzip der Eigenverantwortung und der Überzeugung, dass kollektive Intelligenz zum Finden von nachhaltig wirksamen Entscheidungen einen wertvollen Beitrag leistet.

\section{Drei Projektskizzen}

Die erste der drei ausgewählten Skizzen heisst: «Was tun? Umgang mit psychisch belasteten Menschen in Schule und Betrieb" Dieses Projekt setzt sich zum Ziel, in einem zweiteiligen Seminar Vorgesetzte und Personalverantwortliche für den Umgang mit psychisch belasteten Mitarbeitenden zu sensibilisieren und ihnen Kompetenzen für den Umgang zu vermitteln.

Das zweite ausgewählte Projekt «Kantonale Grundversorgung angesichts schwindender Ressourcen und steigendem Bedarf» stellt sich der Herausforderung, Vorschläge für die Sicherstellung einer gut funktionierenden Grundversorgung im Kanton Thurgau zu erarbeiten.

Die dritte Idee mit dem Namen "eMediplan» hat den Anspruch, eine Konzeptskizze für einen elektronisch geführten, vollständigen und aktuellen Medikamentenplan zu erarbeiten.

Im Anschluss an die Tagung überprüft die Projektgruppe, ob die drei favorisierten Projekte den Kriterien des Patientennutzens, der Innovation und der Interdisziplinarität entsprechen. Danach gibt sie grünes Licht für die Vertiefung der Projektideen. Die drei Projektleitungen suchen sich Mitarbeitende, die sich dem Thema widmen wollen, und beginnen mit der Arbeit. An regelmässig stattfindenden Steuergruppensitzungen berichten die drei Leitungen über den aktuellen Stand der Arbeit. Ein Soundingboard gibt den Projektleitungen nach etwa einem halben Jahr ein Feedback und eine Aussensicht.

Wie nicht anders zu erwarten, entwickeln sich die drei Gruppen sehr unterschiedlich. Das Seminarkonzept für den Umgang mit seelisch belasteten Menschen findet grossen Anklang. Die drei ausgeschriebenen Seminare sind im $\mathrm{Nu}$ ausgebucht und erhalten ein positives Feedback von den Teilnehmenden. Eine Besonderheit des Seminarkonzepts, der Einbezug von direkt betroffenen, psychisch kranken Menschen, wird von den Seminarteilnehmenden sehr geschätzt. Die Projektleitung für eine gut funktionierende Grundversorgung sieht sich vor die Herausforderung gestellt, die verschiedenen Perspektiven der beteiligten Leistungserbringer in einer Gesamtschau zu vereinen. Dies erweist sich als anspruchsvolles Unterfangen. Die Projektgruppe entwickelt ein Konzept, wie die Zusammenarbeit zwischen den verschie- 
denen Disziplinen mittels Versorgungsnetzwerken optimiert werden könnte. Ausserdem beschreibt sie bereits existierende, gute Beispiele aus anderen Regionen und regt die verstärkte Nutzung des Informationsportals «Sozialnetz Thurgau» an.

Das Konzept "eMediplan» befasst sich mit dem Missstand, dass die Übersicht über die verordneten Medikamente besonders bei Patienten mit Mehrfachdiagnosen oft zu wünschen übrig lässt. Es gelingt, einen Standard mit einer darauf aufbauenden Papiervariante und einem 2-D-Barcode zu definieren, der allen Beteiligten eine rasche und vollständige Übersicht über die verordneten Medikamente verschafft. Dies funktioniert natürlich nur unter der Voraussetzung, dass alle beteiligten Stellen, die Medikamente verordnen resp. abgeben, sich zuverlässig am Ausfüllen des Medikamentenplans beteiligen! Die von eMediplan vorgeschlagene Lösung ist mit dem geplanten elektronischen Patientendossier von eHealthSuisse kompatibel.

Knapp anderthalb Jahre nach der ersten Tagung findet im November 2014 ein zweites Treffen aller Stakeholder statt. Im Kloster Fischingen im Hinterthurgau berichten die Projektleitungen über die erzielten Ergebnisse. Die Berichte werden in Diskussionsgruppen vertieft. Das Referat von Karin Frick, der Forschungsleiterin des Gottlieb Duttweiler Instituts, über Megatrends im Gesundheitswesen bildet eine geschätzte Bereicherung des Anlasses. Die Berichte und Konzepte der Projektleitungen sowie das Referat von Frau Frick können von der Website des Amtes für Gesundheit Thurgau heruntergeladen werden (www. gesundheit.tg.ch/xml_61/internet/de/application/ f16381.cfm).

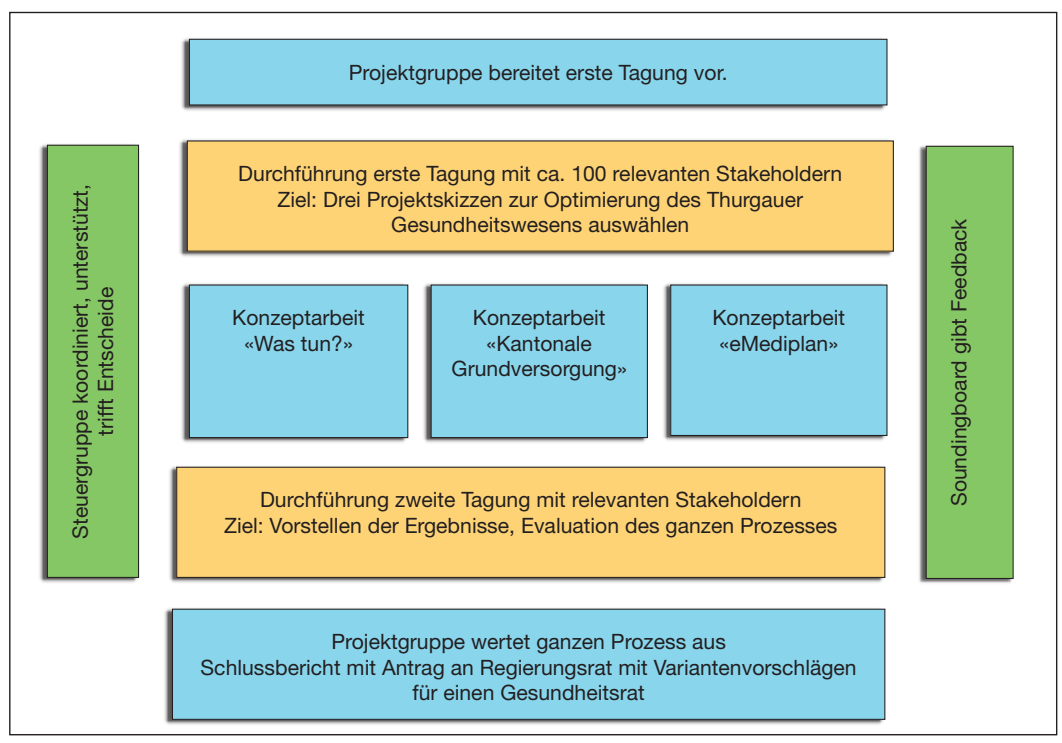

Abbildung 1: Das Vorgehen beim Projekt «Brennpunkte Gesundheit Thurgau» im Überblick.

\section{Ausblick}

Das Seminar «Was tun?» kann in einen Regelbetrieb übergeführt werden und wird nun von der Perspektive Thurgau, einer kantonalen Einrichtung, die sich unter anderem mit Gesundheitsförderung und Prävention befasst, weiterbetreut.

Das Konzept «eMediplan» muss über den Kanton Thurgau hinaus bekannt gemacht und verankert werden. Es braucht eine nationale eMediplan-Kampagne und einen national anerkannten eHealthStandard "eMedikation». Wenn alles plangemäss verläuft, wird im Frühjahr 2016 ein erster Pilotbetrieb, wenn möglich im Kanton Thurgau, gestartet.

Das Thema «Kantonale Grundversorgung» erfordert noch weitere Konkretisierungen. Es braucht einen Träger, der die Vorschläge, die im Abschlussbericht gemacht werden, begleitet und umsetzt. Am sinnvollsten erfolgt dies mit einem Pilotbetrieb in einer Region, in der die Grundversorgung aktuell gefährdet ist. Diesbezügliche Abklärungen sind im Gange.

Wir denken zur Zeit intensiv über die Zukunft des übergeordneten Projekts «Brennpunkte Gesundheit Thurgau» nach. Dabei ist klar, dass ein solches Projekt nur mit der Unterstützung und dem politischen Willen der Regierung weitergeführt werden kann. Ebenso braucht es das Engagement der beteiligten Organisationen. Letzteres ist erfreulicherweise vorhanden: Viele Organisationen haben darauf verzichtet, das ihnen zustehende Sitzungsgeld einzufordern. Sie haben auch die Absicht geäussert, dies bei einer Fortführung des Projekts weiterhin so zu handhaben.

Die Projektgruppe ist momentan damit beschäftigt, die gemachten Erfahrungen in einem Konzept zusammenzufassen. Dieses nimmt die gemachten Erfahrungen und Lerneffekte auf und schlägt vor, wie das Projekt weitergeführt werden soll.

Als zweites Ziel erarbeitet die Gruppe für die Kantonsregierung Variantenvorschläge für einen $\mathrm{Ge}$ sundheitsrat, der dem Departementsvorsteher bei Fragen von strategischer Bedeutung beratend zur Seite steht. Die Regierung wird zu gegebener Zeit darüber befinden, ob und wie ein solcher Rat im Thurgau installiert werden soll.

\section{Schlussbetrachtung}

Das Projekt «Brennpunkte Gesundheit Thurgau» verfolgt zwei Ziele: Es will einen konkreten Beitrag zu einer integrierten Versorgung im Kantonalen Gesundheitswesen leisten. Dieses Ziel ist mit den drei Projekten erfüllt worden. Wie im vorliegenden Bericht gezeigt wurde, haben es Projekte mit einem 
Kernaussagen der am Projekt beteiligten Ärzte zu ..

\begin{tabular}{|c|c|c|}
\hline ... Schlüsselerlebnis & $\begin{array}{l}\text { Aus der «prima materia» entwickelte sich } \\
\text { erstaunlicherweise Konkretes. Niemand } \\
\text { wusste zu Beginn, wohin der Weg führt. } \\
\text { Ein Schlüsselerlebnis war der Workshop } \\
\text { im Juni 2013, wo sich über } 100 \text { Leute Ge- } \\
\text { danken zu Brennpunkten des Gesundheits- } \\
\text { wesens machten. Dies zeigt eindrücklich, } \\
\text { dass es möglich ist, "bottom up» mitein- } \\
\text { ander zu kommunizieren und etwas zu } \\
\text { erarbeiten. }\end{array}$ & $\begin{array}{l}\text { Wir haben einen neuen, aus verwaltungs- } \\
\text { technischer Sicht «ungewohnten» Ansatz } \\
\text { gewählt, indem wir die Partner aus dem } \\
\text { Gesundheitswesen gefragt haben, wo der } \\
\text { Schuh drückt. Die drei gewählten Projekt- } \\
\text { themen haben eine hohe Relevanz auf } \\
\text { kantonaler, mit «eMediplan» sogar auf } \\
\text { nationaler Ebene. }\end{array}$ \\
\hline ... Modellcharakter & $\begin{array}{l}\text { Der Modellcharakter des Projekts ist auf } \\
\text { jeden Fall gegeben. }\end{array}$ & $\begin{array}{l}\text { Die Vernetzung unter den Leistungs- } \\
\text { erbringern im Gesundheitswesen wurde } \\
\text { gestärkt. Elemente sind: sich kennen - } \\
\text { sich austauschen - zusammenarbeiten - } \\
\text { Vertrauen bilden. }\end{array}$ \\
\hline ... Grenzen des gewählten Ansatzes & $\begin{array}{l}\text { Es gibt hauptsächlich zwei Grenzen: die } \\
\text { Finanzen und die Herausforderung, genü- } \\
\text { gend (qualifizierte) Leute zu finden, die } \\
\text { bereit sind, an den Projekten mitzuarbei- } \\
\text { ten. }\end{array}$ & $\begin{array}{l}\text { Schlüsselelement war die Rolle des Pro- } \\
\text { jektleiters, der Projektleiterin. Diese müs- } \\
\text { sen einerseits die finanziellen, vor allem } \\
\text { aber auch die zeitlichen Ressourcen zur } \\
\text { Verfügung stellen, damit die Projekte } \\
\text { erfolgreich abgewickelt werden können. }\end{array}$ \\
\hline ... Was lernt man für die Zukunft? & $\begin{array}{l}\text { Soll das ursprüngliche Ziel des Projekts - } \\
\text { eine nachhaltige Konfliktlösung - erreicht } \\
\text { werden, ist Transparenz ein absolutes } \\
\text { Muss. }\end{array}$ & Dem schliesse ich mich an. \\
\hline
\end{tabular}

hohen Praxisbezug einfacher, relativ schnell eine konkrete Wirkung für die Bevölkerung zu entfalten. Eher theoretische Themen wie «Kantonale Grundversorgung" oder "eMediplan" haben aber durchaus auch ihre Berechtigung. Sie können, wenn sie bis zur Umsetzung vorangetrieben werden, eine grosse Hebelwirkung entfalten. Dies braucht aber mehr Zeit als die anderthalb Jahre, die uns zur Verfügung standen. Die Projektgruppe war sich aber in der Schlussevaluation einig, dass es beide Projekttypen braucht: solche, bei denen eine rasche Umsetzung möglich ist aber auch solche, die mehr Zeit benötigen, bis sie zur Realisierung gelangen.
Neben den Ergebnissen, die aus der konkreten Projektarbeit heraus entstanden sind, hat «Brennpunkte Gesundheit Thurgau» noch ein anderes, «immaterielles» Resultat gezeitigt. Es hat Menschen zusammengebracht, die sonst nicht miteinander in Kontakt gekommen wären. Dieser Aspekt wurde in vielen wertschätzenden Zuschriften bestätigt. Diese Vernetzung, die an den Tagungen oder in der konkreten Konzeptentwicklung erfolgt ist, macht es einfacher, bei zukünftigen Problemstellungen aufeinander zuzugehen und gemeinsam unbürokratische Lösungen zu finden. Zum Schluss möchte ich allen Beteiligten für die intensive, lehrreiche und gewinnbringende Zusammenarbeit danken! 